\title{
Comparison of short courses of oral prednisolone and fluticasone propionate in the treatment of adults with acute exacerbations of asthma in primary care
}

\author{
Mark L Levy, Craig Stevenson, Toni Maslen
}

\begin{abstract}
Background - Oral corticosteroids used in short courses for acute asthma are regarded as safe, although the frequent use of these drugs may result in patients suffering from systemic side effects. It has become common practice for patients to increase their own inhaled corticosteroid intake when their asthma goes out of control, but it has never been established whether a high dose of inhaled corticosteroid can be as effective as a short course of oral corticosteroid in the treatment of acute exacerbations.
\end{abstract}

Methods - A multicentre, randomised, double blind, double dummy, parallel group study was undertaken to determine whether the introduction of a high dose of inhaled fluticasone propionate $(2 \mathrm{mg}$ daily) is as effective as a short reducing course of oral prednisolone (starting at $40 \mathrm{mg} /$ day and reducing by $5 \mathrm{mg}$ every other day) in the treatment of acute exacerbations of asthma not considered severe enough for admission to hospital but requiring treatment with oral corticosteroid.

Results - Four hundred and thirteen adult asthmatic subjects who presented to their general practitioner with an acute exacerbation of asthma were recruited in 47 general practices in the United Kingdom. Treatment failures, defined as a reduction in peak expiratory flow (PEF) to below $60 \%$ of the patient's best/predicted value on two consecutive occasions or persistent symptoms with no improvement on three consecutive days, occurred in $23 \%$ of patients who received oral prednisolone and $27 \%$ who received inhaled fluticasone propionate (difference in percentage of treatment failures $4.3,95 \%$ CI -4.1 to $12.8, p=0.31$ ). In each group $48 \%$ were classified as treatment successes, defined as a $10 \%$ or greater increase in percentage best/predicted morning PEF. Both treatments were equally well tolerated.

Conclusions - There is no evidence of a significant difference in efficacy between a reducing dose course of oral prednisolone and high dose inhaled fluticasone propionate in mild exacerbations of asthma which do not require admission to hospital.

(Thorax 1996;51:1087-1092)
Keywords: asthma, steroids, fluticasone propionate, general practice.

Corticosteroids are the most effective anti-inflammatory therapy available for the treatment of asthma. Current asthma management guidelines recommend that inhaled corticosteroids are introduced at a very early stage in the treatment of all but the mildest of asthmatics. ${ }^{1}$ These guidelines also recommend the use of oral corticosteroids in the treatment of acute attacks of asthma. There is good evidence to suggest that oral corticosteroids prevent the progression of exacerbations, decrease the need for admission to hospital, and reduce morbidity due to asthma in adults ${ }^{2}$ and children. ${ }^{3}$ It is questionable whether frequent short courses of oral corticosteroids may result in the systemic side effects with which these drugs are associated such as suppression of the hypothalamic-adrenal axis and osteoporosis. ${ }^{4}$

It is recognised that in chronic adult asthma inhaled steroids may help to reduce long term oral corticosteroid use $\mathrm{e}^{5-7}$ and to improve lung function with a less pronounced effect on adrenocortical action when used instead of or combined with oral prednisolone. ${ }^{8}$ Previous studies have demonstrated the benefits of patients increasing their own inhaled corticosteroid intake when peak expiratory flow rates fall below a critical level through self-management plans discussed with their own physicians. ${ }^{9}$ However, it has never been established whether a high dose of an inhaled corticosteroid can be as effective as a short course of oral corticosteroid in the treatment of acute exacerbations.

We have therefore examined whether the introduction of a high dose of inhaled fluticasone propionate ( $2 \mathrm{mg}$ daily) could be as effective as a short reducing course of oral corticosteroid in the treatment of acute exacerbations of asthma which were not considered severe enough to necessitate admission to hospital but which did, in the opinion of the general practitioner, require treatment with oral corticosteroid.

\section{Methods}

The randomised, double blind, double dummy, parallel group study was conducted in 74 general practice centres throughout the UK between June 1993 and June 1994. The study was conducted in accordance with good clinical practice guidelines issued by the European Commission in $1990^{10}$ and with the Declaration 
of Helsinki. Approval was obtained from the Royal College of General Practitioners (RCGP) research ethics committee and the appropriate local research ethics committees.

Adult asthmatic patients were considered eligible for entry into the study if they presented to their GP with a mild exacerbation of asthma which, in the clinician's opinion, was not severe enough to necessitate admission to hospital but which did require treatment with a short course of oral corticosteroid. Patients were only entered into the study if their peak expiratory flow (PEF) measured immediately on presentation, or one hour after any acute therapy with a bronchodilator where appropriate, was at least $60 \%$ of best or, where not available, $60 \%$ of the predicted ${ }^{11}$ value, but no greater than $90 \%$ of best/predicted - this PEF value is referred to as the entry PEF throughout the remainder of this report. Suitable patients were then randomised in a double blind fashion by the general practitioner, in balanced blocks of six, to receive either inhaled fluticasone propionate $1 \mathrm{mg}$ twice daily via a Volumatic or a reducing course of oral prednisolone (starting at $40 \mathrm{mg}$ and reducing by $5 \mathrm{mg}$ every two days) for a period of 16 days. All concurrent asthma medications, including existing inhaled corticosteroid therapies and non-asthma medications, were continued throughout the study.

On entry to the study demographic details and asthma history were recorded. After checking inhaler technique, patients were given the first dose of both oral and inhaled study medication on day 1 before leaving the surgery. The second daily dose of inhaled study medication was taken by the patients before retiring to bed that evening. During the first week of the study patients returned to the doctor's surgery every two days to be examined. All patients kept a daily record booklet in which use of the study medications was recorded. An asthma symptom score, morning and evening PEF (best of three using a mini-Wright PEF meter prior to bronchodilator medication), and the need to use the rescue medication provided (oral prednisolone $40 \mathrm{mg}$ ) in case of treatment failure were also recorded daily. At each visit the physician recorded changes in medication, intercurrent illness, and adverse events.

The primary outcome measure was "treatment failure". A patient was categorised as a treatment failure if (a) PEF fell below $60 \%$ of the best/predicted value on two consecutive occasions (for example, morning and evening or vice versa), or (b) a symptom score of 3 was recorded on three or more consecutive days (a score of 3 indicated that the symptoms were the same as or worse than on entry to the study), or (c) the patient withdrew from the study because of uncontrolled symptoms or an adverse event related to asthma. Patients defined as treatment failures were withdrawn from the study and given appropriate additional treatment.

The intended power of the study was $80 \%$ (at the 5\% significance level) to detect a difference in the proportion of treatment failures between treatments of 10 percentage points or greater, assuming a failure rate of $20 \%$ in the oral prednisolone group and a higher rate of failure in the fluticasone propionate group. The failure rate and its significance was a clinical judgement and, in consultation with the ethics committee of the RCGP, it was agreed that a difference of 10 percentage points or more in the failure rate between the two groups would be of clinical significance. Based on these criteria a total of 582 patients was required. However, this was a difficult population of patients to recruit and the study was ended when a total of 413 patients was recruited.

The difference in the proportion of treatment failures was analysed using a test based on the normal approximation of the binomial distribution. A logistic regression analysis was performed to assess the relationship of the three factors: treatment, patient's entry PEF, and existing dose of inhaled corticosteroid on entry, with the dependent variable, treatment failure (yes/no). The statistical package used in the analysis was GLIM V4.0 for PC Windows.

Any patient not categorised as a treatment failure, who demonstrated an improvement of at least $10 \%$ in percentage best/predicted morning PEF during the course of the 16 day treatment period from day 2 (the first available morning PEF value), was classified as a treatment success.

The daily asthma symptom scores and the changes seen in the mean diurnal variation in PEF were examined. Diurnal variation was calculated as the percentage amplitude of the mean. ${ }^{12}$ Where medians are quoted in the text, the adjacent figures in brackets represent the lower and upper quartiles (q1;q3). All analyses were performed on an intention-to-treat basis. For evaluable patients not completing the study, their data were included in the analyses up until they withdrew. Statistical comparisons were based on two sided tests.

\section{Results}

Seventy four centres were set up but only 47 of these recruited the 413 patients randomised to treatment (median five patients $(3 ; 10)$ per centre). The randomisation process was successful in providing well matched groups of patients for age, height, and sex, as well as duration/severity of asthma and entry dosages of inhaled corticosteroid medication (table 1). Six patients in the fluticasone group and four in the prednisolone group were not considered evaluable for the treatment failure and success criteria, either because they were withdrawn in error by the investigator (seven patients) or because they did not complete at least 12 days of the study (three patients). The total number of evaluable patients in each group was 200 in the fluticasone group and 203 in the prednisolone group.

\section{TREATMENT OUTCOME}

Treatment failures

Fifty four patients $(27 \%)$ who received inhaled fluticasone propionate were categorised as treatment failures compared with $46(22.7 \%)$ who received a reducing course of oral pred- 
Table 1 Baseline patient characteristics

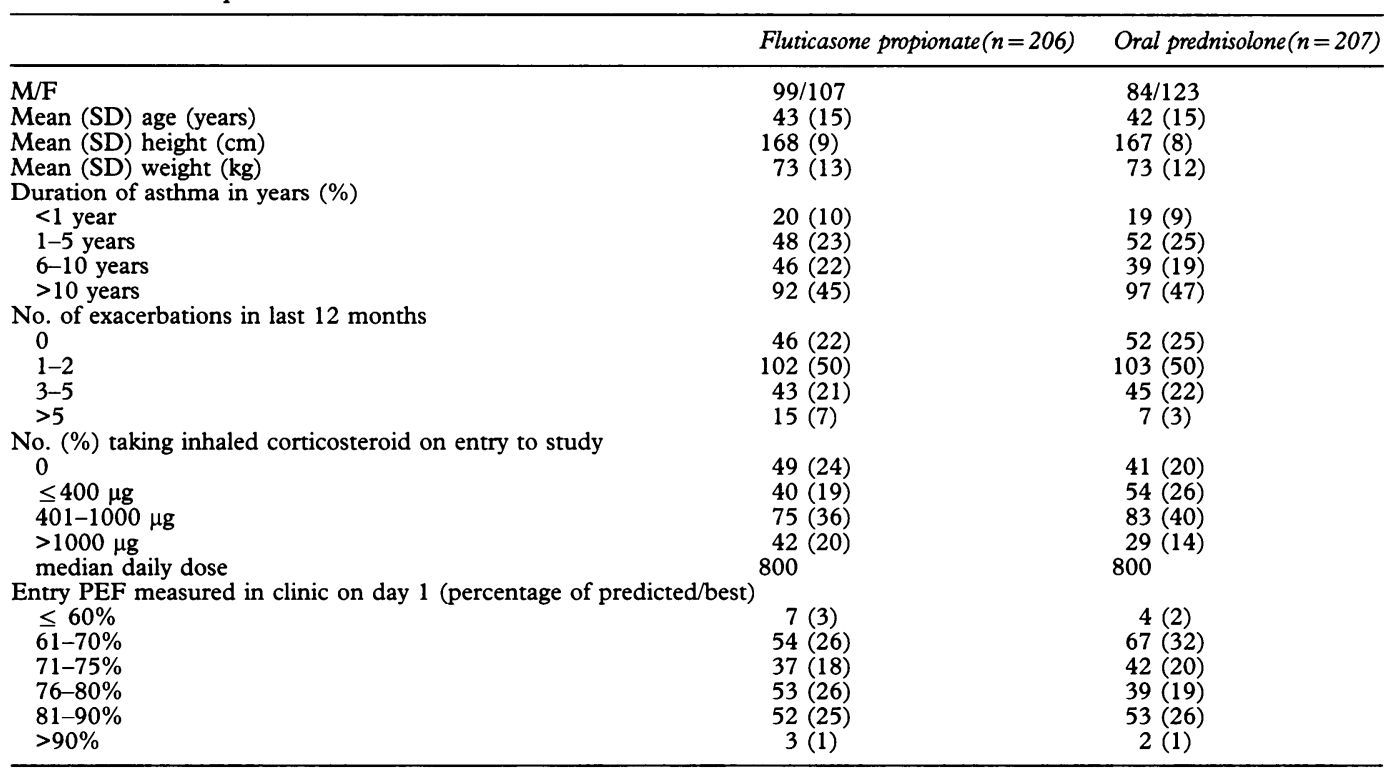

nisolone (fig 1). The difference in the proportion of treatment failures was 4.3 percentage points $(95 \%$ confidence interval -4.1 to 12.8 , $\mathrm{p}=0.31$ ). A logistic regression analysis con-

A

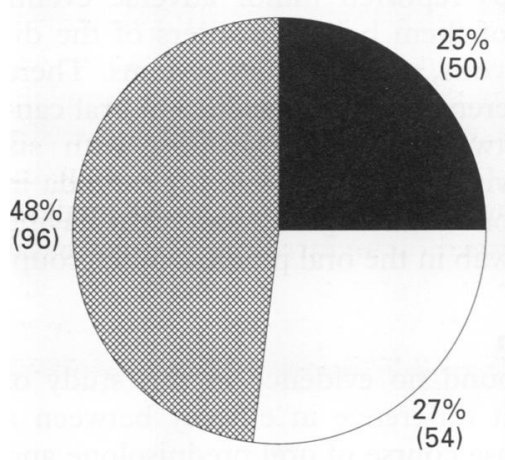

B

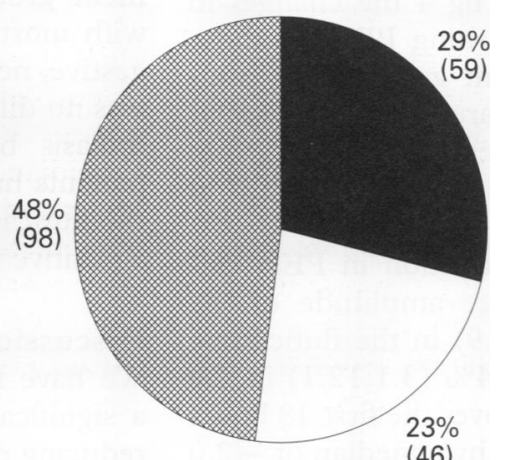

(46)

Treatment failures

Treatment successes

Neither

Figure 1 Proportion of treatment failures and successes with $(A)$ fluticasone propionate $(n=200$ evaluable $)$ and $(B)$ prednisolone $(n=203$ evaluable $)$.

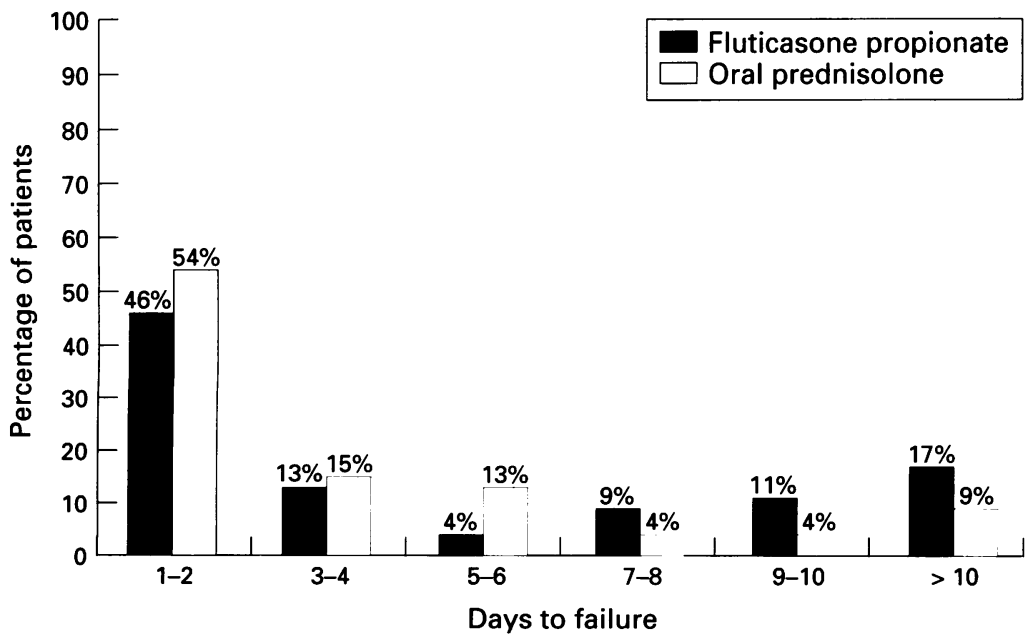

Figure 2 Distribution of time to treatment failure for patients classified as a "treatment failure" ( $n=54$ on fluticasone and $n=46$ on oral prednisolone). firmed that a comparison of treatment failure, overall and between treatment groups, was independent of the patient's entry PEF, expressed as a percentage of best/predicted, and the existing dose of inhaled corticosteroid on entry. The reasons for treatment failure were similar in the two treatment groups with most being due to reductions in PEF or failure to improve symptoms.

In the group of patients defined as treatment failures the median number of days to failure was three days $(1 ; 9)$ in the fluticasone group and two days $(1 ; 5)$ in the prednisolone group. The distribution of the times to treatment failure for each of the two groups can be seen in fig 2.

\section{Treatment successes}

Patients categorised as treatment failures could not be categorised as treatment successes. In both treatment groups $48 \%$ of patients had an improvement of at least $10 \%$ in best/predicted morning PEF and were categorised as treatment successes (fig 1 ). The median time taken to achieve success was three days $(1 ; 5)$ in the fluticasone group and three days $(2 ; 6)$ in the prednisolone group.

\section{Neither}

Twenty nine per cent of patients treated with oral prednisolone and $25 \%$ treated with fluticasone propionate did not meet the study definition of either a treatment success or failure.

The mean percentage predicted entry PEF was similar for patients in all of the outcome groups with mean (SD) values of $74(8.4) \%$, $75(8) \%$, and $76.3(8) \%$ in the "treatment failure", "treatment success", and "neither" groups, respectively. The median dose of inhaled corticosteroid at study entry in these three groups was $800 \mu \mathrm{g}(400 ; 1000 \mu \mathrm{g}), 600 \mu \mathrm{g}$ $(0 ; 1000 \mu \mathrm{g})$, and $800 \mu \mathrm{g}(400 ; 1000 \mu \mathrm{g})$, respectively. 


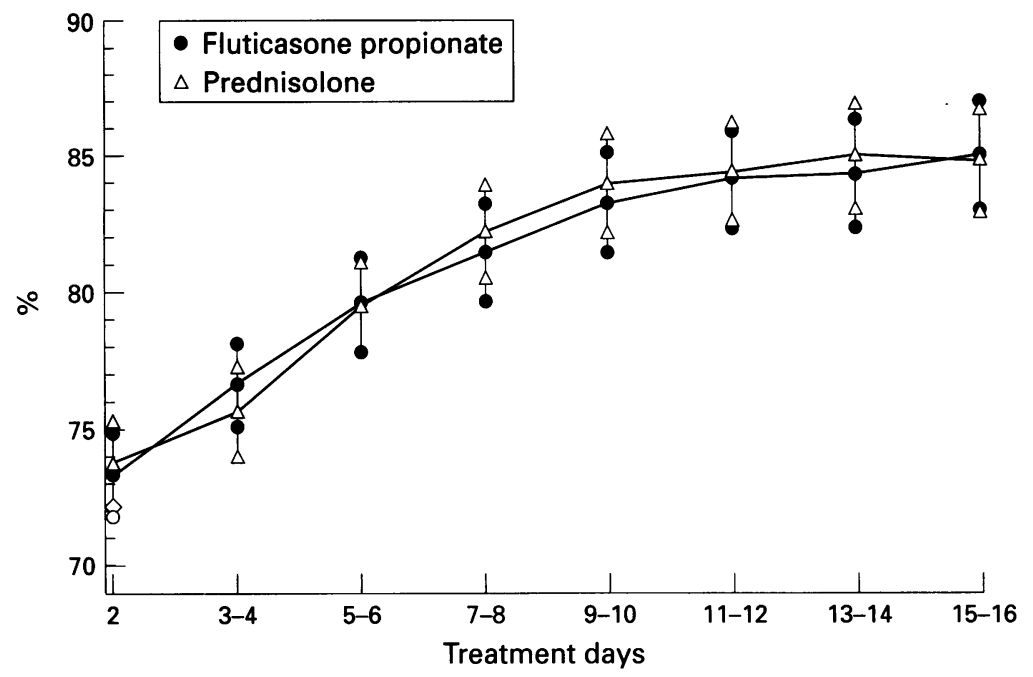

Figure 3 Treatment profiles of mean (95\% confidence intervals) percentage predicted morning PEF of total sample. Note: the mean values are calculated from the data of patients still in the study at that time point: fluticasone propionate $(n=204$ day 2 , $n=162$ day 15/16); prednisolone ( $n=203$ day $2, n=165$ day $15 / 16)$.

\section{LUNG FUNCTION}

Mean morning PEF, expressed as a percentage of best/predicted, increased at a similar rate over the 16 day study period in both treatment groups. The mean levels in percentage predicted morning PEF over the treatment period can be seen in fig 3 . In fig 4 the changes in percentage predicted morning PEF for those patients in both treatment groups who completed the study period are shown. A similar proportion of patients in each treatment group was above and below $85 \%$ predicted PEF at the end of the study.

The median diurnal variation in PEF, calculated as the percentage amplitude of the mean, was $7.5 \%(2.7 ; 12.9)$ in the fluticasone propionate group and $6.4 \%(3.1 ; 12.1)$ in the oral prednisolone group over the first 48 hours of the study. It decreased by a median of -2.0

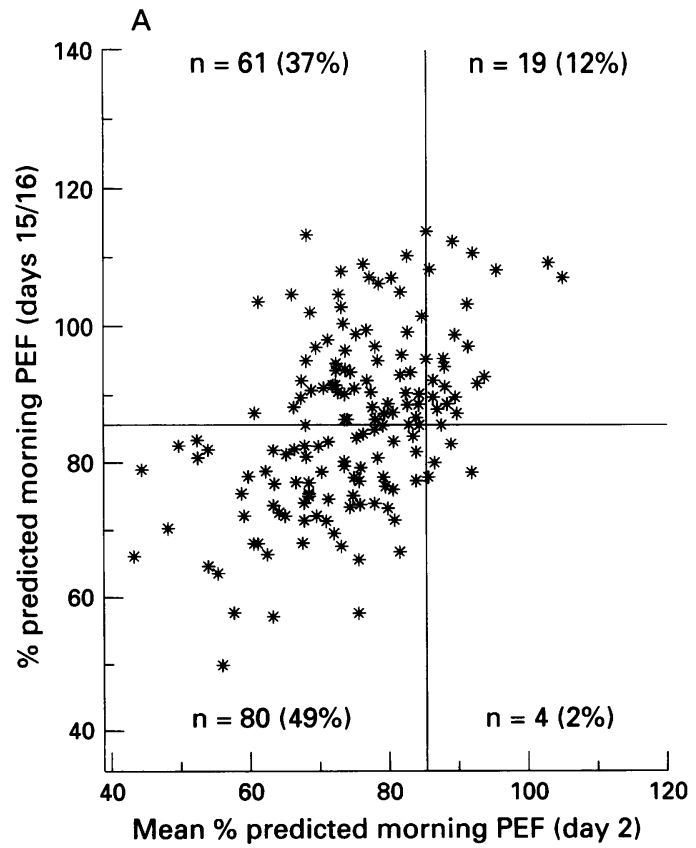

percentage points $(-8.8$ to 2.4$)$ in the fluticasone group and by -2.1 percentage points ( -5.6 to 2.3$)$ in the oral prednisolone group over the course of the study.

\section{SYMPTOMS}

The median symptom score recorded by patients in each treatment group reduced from $3(2 ; 3)$ on entry to the study to $1(0 ; 2)$ by the end of treatment.

\section{ADVERSE EVENTS}

Both treatment regimens appeared to be equally well tolerated. Five patients reported serious adverse events during the study, three of whom were receiving inhaled fluticasone propionate. These events included one fatality due to a cardiac arrest resulting from coronary artery atheroma. The investigator deemed this death to be unrelated to the study medication. The other two events in this group were a reexacerbation of asthma and a report of a serious headache. The two serious events reported by patients receiving oral prednisolone included a re-exacerbation of asthma and a report of shortness of breath. None of these five serious events was thought to be likely to be related to the study drug.

Forty four per cent of patients in both treatment groups reported minor adverse events with most of them being disorders of the digestive, nervous, or respiratory systems. There was no difference in the incidence of oral candidiasis between the two groups, with six patients having a positive swab for candida in the fluticasone treated group and eight having a positive swab in the oral prednisolone group.

\section{Discussion}

We have found no evidence in this study of a significant difference in efficacy between a reducing dose course of oral prednisolone and

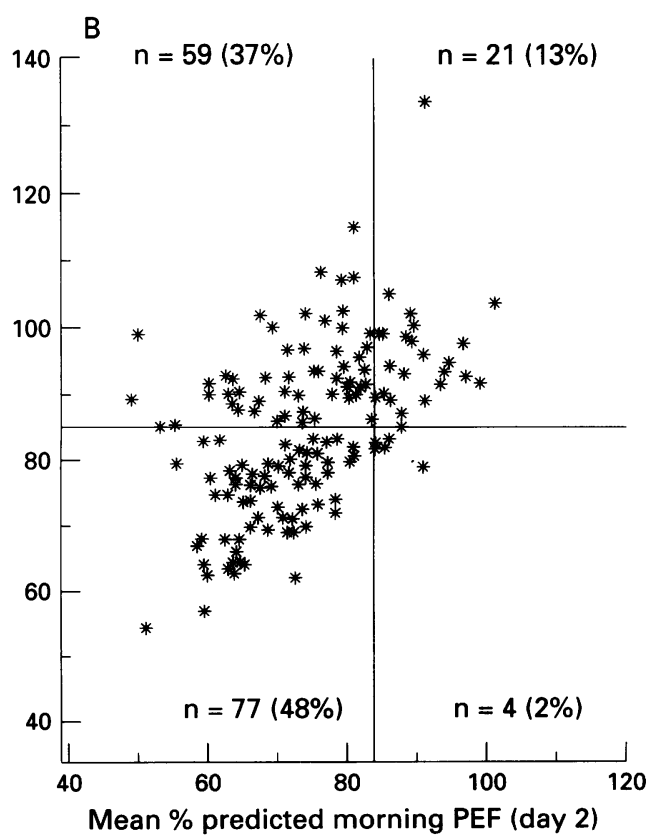


high dose inhaled fluticasone propionate in mild exacerbations of asthma which do not require hospital admission. Whether inhaled fluticasone propionate could be as effective as oral prednisolone in the treatment of more severe exacerbations, either in primary care or hospital, needs further investigation.

This study was unable to show any significant differences in clinical outcome between the two treatment groups. This may have been due to the reduced power of the study which arose from the difficulty experienced in recruiting patients. In the event we have presented data on 403 (evaluable) patients rather than the intended 582, and although this is still a large sample of patients in which to investigate the outcome, we estimate the final power to detect our defined outcomes at the $5 \%$ level to be of the order of $57 \%$. We also acknowledge that the observed difference in the proportions of treatment failures, which is accepted as the best estimate of the true treatment difference, ${ }^{13}$ favoured oral prednisolone though this difference in treatment failure was 4.3 percentage points which is well below our predefined minimum clinical difference of 10 percentage points. The proportion of successes was similar with both treatments.

Oral corticosteroids are widely accepted as the treatment of choice in acute exacerbations of asthma ${ }^{1}$ and their efficacy is proven. ${ }^{23}$ However, they have been shown to have significant systemic activity. Following long term treatment, systemic side effects with oral corticosteroids can include weight gain, adrenocortical suppression, skin thinning with easy bruising, and osteoporosis. ${ }^{14-16}$ The cumulative effects of repeated short courses of oral corticosteroid may make asthmatic patients susceptible to these potential effects. The topical nature of inhaled corticosteroids limits this potential. Noonan et $a l^{6}$ demonstrated a reduction in oral steroids to zero in approximately $75 \%$ of patients following the introduction of inhaled fluticasone propionate compared with only $3 \%$ of patients given placebo. In the same study the number of patients with abnormal plasma cortisol levels following the introduction of inhaled fluticasone propionate was lower than in the placebo group. These results ${ }^{6}$ suggest that both fluticasone propionate and prednisone can be effective treatments for asthma and that the incidence of systemic side effects is reduced following replacement of oral steroid by inhaled fluticasone propionate. Using a high dose of an inhaled corticosteroid instead of a course of oral corticosteroids in the treatment of acute exacerbations of asthma may therefore be safer in the long term with respect to systemic side effects, although our study has not specifically addressed this issue.

When designing this study one difficulty faced was the selection of the oral corticosteroid regimen to be used. Canvassing of general practitioners indicated a wide variation in the dosages of oral corticosteroids prescribed and their duration of use. Although recent data suggest that there is no additional benefit from tapering oral steroids when treating acute exacerbations of asthma, ${ }^{17}$ the oral steroid re- gimen selected for this study was representative of the clinical practice at the time the study was designed. The reducing course chosen should have provided sufficient steroid cover to achieve a clinical benefit, particularly in patients who continued to take their existing inhaled corticosteroid therapy. The BTS guidelines on the management of acute asthma ${ }^{1}$ recommend the use of 30-60 mg of prednisolone daily for 1-3 weeks after an asthma attack. In our study patients were prescribed at least $30 \mathrm{mg}$ a day for six days. The failure of the oral prednisolone regimen to improve percentage best/predicted PEF to above $85 \%$ by day 16 in over $50 \%$ of our patients who completed the study may support Webb's conclusion that oral prednisolone should be prescribed at a dose of $0.6 \mathrm{mg} / \mathrm{kg}$ body weight (that is, $40 \mathrm{mg}$ daily in a $70 \mathrm{~kg} \mathrm{man}$ ) for at least two weeks following an acute asthma attack. ${ }^{18}$

About half of the patients who were defined as treatment failures failed to respond to treatment within the first two days $(46 \%$ in the fluticasone propionate group and $54 \%$ in the prednisolone group; fig 2). For those patients who "failed" the median time to treatment failure was three days in the fluticasone propionate group and two days in the prednisolone group; some patients took up to 10 days to be defined as treatment failures. These data are consistent with a study conducted by Bucknall et al which showed that a significant proportion of patients were still suffering from symptoms 13 days after being discharged from hospital following an acute asthma attack. ${ }^{19}$ Levy et al also found that, in $66 \%$ of patients on whom data were available, PEF variation was greater than $15 \%$ two months after attending hospital for an acute exacerbation of asthma. ${ }^{20}$ These data suggest that, after initial treatment for acute asthma (hospital or community), patients should be seen frequently - we suggest daily for the first three days then weekly for at least four weeks or until the clinical signs and symptoms have resolved.

It could be argued that the early withdrawal (median 2 days) of patients from the study because of treatment failure meant they were not given enough time to respond to treatment. However, this was a double blind, double dummy study and therefore we felt ethically obliged to withdraw these patients for safety reasons. The median time to withdrawal was similar irrespective of the treatment group, so this study criterion did not unbalance the study in terms of the conclusions.

The severity of the asthma exacerbations reported can be defined in terms of PEF expressed as a percentage of best/predicted value. For the purpose of this study the first PEF recorded was measured after acute treatment with a bronchodilator (entry PEF) where this was indicated. The use of the entry PEF (one hour after bronchodilator) prevents an assessment of the true severity of the exacerbation on presentation to the surgery. Approximately $50 \%$ of the patients entered into the study had an entry PEF that ranged from $50 \%$ to $75 \%$ of the best/predicted value. The BTS guidelines for the management of asthma recommend that 
oral prednisolone be given to patients whose PEF lies between $50 \%$ and $75 \%$ of predicted or best, 15-30 minutes after nebulised bronchodilator. ${ }^{1}$ Half of the patients recruited into the study did meet this criterion, but the only guidance given to doctors participating in the study was that, in their opinion, patients should require a course of oral corticosteroids to be considered for inclusion. The number of patients entered into the study whose entry PEF was greater than $75 \%$ of best/predicted indicates that PEF is not the only criterion used by general practitioners to assess whether courses of oral corticosteroids are indicated. Whilst most asthma attacks are treated by general practitioners ${ }^{2122}$ there is wide variation in the treatment provided, often at variance with recommended guidelines. In one study reported by Neville et $a l^{22} 56 \%$ of asthmatic patients suffering acute attacks of asthma were treated with systemic corticosteroids; their clinical severity ranged from "not breathless" to "too breathless to talk". Our study suggests that general practitioners use other parameters such as clinical signs and patient's previous history in addition to lung function when deciding when to initiate courses of oral corticosteroids. Furthermore, our results provide some guidance for general practitioners in deciding whether to prescribe an oral or inhaled steroid for the management of mild exacerbations of asthma. We suggest that, if the PEF on presentation or after treatment with bronchodilator therapy is above $60 \%$ best or predicted, high dose inhaled steroids may be used instead of oral steroids.

Diurnal variation in PEF is sometimes used as an indicator of instability of asthma. In this study the diurnal variation in PEF measured in both treatment groups was less than $8 \%$ during the first 48 hours of acute treatment. This low value in itself would not be indicative of a lack of asthma control requiring treatment with oral corticosteroids, so based on the diurnal variation alone very few of these patients would have been recruited into this study. However, it is important to note that both treatments did reduce the median diurnal variation by about two percentage points whilst increasing percentage predicted morning PEF by an average of 11 percentage points.

In conclusion, we have found no evidence of a significant difference in efficacy between a reducing dose course of oral prednisolone and high dose inhaled fluticasone propionate in mild exacerbations of asthma. However, we acknowledge that prednisolone produced a slightly lower proportion of treatment failures and that the power of our study was low. We are unable to comment on the relative profile of unwanted effects from these alternative therapies.

We thank the participating general practitioners for their excellent co-operation with this study which was sponsored by Glaxo Pharmaceuticals UK Ltd.

1 British Thoracic Society. Guidelines on the management of asthma. Thorax 1993;48(Supplement):S1-24.

2 Chapman KR, Verbeek PR, White JG, Rebuck SA. Effect of a short course of prednisolone in the prevention of early relapse after the emergency room treatment of acute asthma. N Engl f Med 1991;324:788-94.

3 Connett GJ, Warde C, Wooler E, Lenney W. Prednisolone and salbutamol in the hospital treatment of acute asthma. Arch Dis Child 1994;70:170-3.

4 Smith N. Corticosteroids and osteoporosis. Thorax 1990; 45:573-8.

5 Laursen LC, Tandorf E, Weeke B. High dose of inhaled budesonide in the treatment of severe steroid dependent asthma. Eur F Respir Dis 1989;140:167-71.

6 Noonan MJ, Chervinsky P, Busse W, Weisberg SC, Pinnas J, Bennett PD, et al. Fluticasone propionate reduces oral prednisolone use while it improves asthma control and quality of life. Am $\mathcal{F}$ Respir Crit Care Med 1995;152: 1467-73.

7 Bosman HG, van Uffelen R, Tamminga JJ, Paanakker LR.Comparison of inhaled beclomethasone dipropionate $1000 \mathrm{mcg}$ twice daily and oral prednisone $10 \mathrm{mg}$ once $1000 \mathrm{mcg}$ twice daily and oral prednisone $10 \mathrm{mg}$
daily in asthmatic patients. Thorax 1994;49:37-40.

8 Toogood JH. Efficiency of inhaled versus oral steroid treatment of chronic asthma. N Engl Regional Allergy Proc 1987; 8:98-103.

9 Beasley R, Cushley M, Holgate ST. A self management plan in the treatment of adult asthma. Thorax 1989;44 $200-4$.

10 CPMP Working Party on efficacy of medicinal products, notes for guidance (111/397/88-EN). Good clinical practice for trials on medicinal products in the European Community. for trials on medic.

11 Nunn AJ, Gregg I. New regression equations for predicting peak expiratory flow in adults. BMf 1989;298:1068-90.

12 Higgins BG, Britton JR, Chinn S. The distribution of peak expiratory flow variability in a population sample. Am Rev Respir Dis 1989;140:1368-72.

13 Gardener MJ, Altman DG. Statistics with confidence confidence intervals and statistical guidelines. BMF 1990: 4.

14 Reid IR. Pathogenesis and treatment of steroid osteoporosis (review). Clin Endocrinol 1989;30:83-103.

15 Rook A, Wilkinson DS, Ebling FJE, Champion RH, Burton JL eds. Glucocorticosteroid induced atrophy. In: Textbook of dermatology. London: Blackwell Scientific, 1986:1814.

16 Seale JP, Compton MR. Side-effects of corticosteroid agents. Med $\mathscr{f}$ A ust 1986;144:139-42.

17 O'Driscoll BR, Kalra S, Wilson M, Pickering CAC, Caroll KB, Woodcock AA. Double-blind trial of steroid tapering in acute asthma. Lancet 1993;341:324-7.

18 Webb JR. Dose response of patients to oral corticosteroid treatment during exacerbations of asthma. BMF 1986; 292:1045-7.

19 Bucknall CE, Robertson C, Moran F, Stevenson RD. Management of asthma in hospital: a prospective audit.BMF 1988;296:1637-9.

20 Levy ML, Robb M, Bradley JL, Winter RJD. Presentation and management of acute asthma across the communityhospital interface. Thorax 1993;48:460-1.

21 Jobanputra $P$, Ford A. Management of acute asthma attacks in general practice. Brf Gen Pract 1991;41:410-3.

22 Neville RG, Clark RC, Hoskins G, Smith B. National asthma attack audit 1991-2. BMf 1993;306:559-62. 